\title{
Antidepressants may lead to a decrease in niacin and NAD in patients with poor dietary intake
}

\author{
Margaretha Viljoen ${ }^{a}$, Annie Swanepoel ${ }^{b}$, Priyesh Bipath ${ }^{c}$ \\ a Department of Psychiatry, School of Medicine, Faculty of Health Sciences, University of Pretoria, South Africa \\ ${ }^{b}$ Hertfordshire Partnership University NHS Foundation Trust, United Kingdom \\ ${ }^{c}$ Department of Physiology, School of Medicine, Faculty of Health Sciences, University of Pretoria, South Africa
}

Corresponding Author:

Prof Margaretha Viljoen (PhD, PhD)

Department of Psychiatry, School of Medicine; Faculty of Health Sciences University of Pretoria, South Africa

E-mail: mviljoen@webafrica.org.za

Tel: +270123293090

\begin{abstract}
The term niacin is the generic name for the two compounds nicotinic acid and nicotinamide, the major dietary precursors for two important coenzymes, nicotinamide adenine dinucleotide (NAD) and its phosphorylated form, NADP. Niacin is important for the maintenance of cellular integrity and energy production and is involved in more than $\mathbf{5 0 0}$ intracellular reactions. Deficiencies of niacin may contribute to neuropsychiatric and neurodegenerative disorders. Patients who develop nutritional deficiencies as a result of poor dietary intake, could potentially suffer from niacin deficiency and NAD depletion. However, de novo synthesis of niacin and NAD in the kynurenine pathway of tryptophan metabolism may compensate for impaired dietary intake. The rate of synthesis of NAD and niacin from tryptophan oxidation depends on the induction of the enzyme indoleamine 2,3dioxygenase (IDO) by pro-inflammatory cytokines such as interferon-gamma. Niacin
\end{abstract}


synthesis is not limited by a decrease in tryptophan and excessive IDO activity may therefore lead to a decline in tryptophan levels. Antidepressants have an anti-inflammatory effect, including reduction of interferon-gamma and therefore inhibition of IDO, the ratelimiting enzyme of the kynurenine pathway. In theory, this could account for increased serotonin as more tryptophan becomes available for serotonin synthesis. However, the downside may be that less NAD and niacin are synthesised downstream, which could exacerbate common psychiatric problems. It is our hypothesis that patients with poor dietary intake, who are treated with antidepressants, are at risk of developing niacin/NAD deficiency with possible development of associated neuropsychiatric symptoms. We therefore propose that niacin supplementation be considered in patients with inadequate diets who are treated with antidepressants. We believe that if this does not happen, a subclinical niacin deficiency may result, which would be difficult to detect as it would cause the same symptoms of the original illness (e.g. depression). Niacin deficiency should be considered and ruled out in all patients with treatment-resistant depression, who have a poor response to antidepressants. This is potentially a cost-effective and easy intervention, which could be examined in a randomized controlled trial.

\section{Introduction}

The term niacin is the generic name for the two compounds nicotinic acid and nicotinamide, the major dietary precursors for two important coenzymes, nicotinamide adenine dinucleotide (NAD) and its phosphorylated form, NADP [1,2]. Niacin is biosynthetically converted to NAD, an acceptor of hydride equivalents, to form the reduced nucleotide NADH. NAD and its phosphorylated and reduced forms, NADP, NADH and NADPH are essential for the maintenance of cellular integrity and energy production and are said to be 
involved in more than 500 intracellular reactions. NAD serves as cofactor in oxidationreduction reactions and as substrate in important biochemical reactions such as mono- and poly-ADP-ribosylation, protein deacetylation, and ADP-ribose cyclization [3]. NAD is the substrate for the DNA nick sensor, poly (ADP-ribose) polymerase (PARP) which is essential for the repair of DNA damaged by reactive oxygen species. In addition, NAD serves as substrate for sirtuins (silent information regulator of gene transcription), a class of enzymes shown to play a role in gene silencing and said to be of importance for longevity. Another far-reaching effect of NAD is through the NAD-dependent ADP-ribose cyclization that produces mediators of calcium signalling [3-5].

Deficiencies or disturbances in the levels of NAD or its phosphorylated or reduced forms, have been implicated in many diseases, including neurodegenerative disorders $[5,6]$. Indications are that supplementation of NAD, often in the form of niacin, can protect neurons from degeneration caused by mechanical and neurotoxic injury, autoimmune activity and ischaemic damage, and that it may have beneficial effects in conditions such as Parkinson's disease, autism, chronic fatigue syndrome and possibly major depression and anxiety $[3,5,7,8]$.

Niacin deficiency, and by implication NAD depletion, may develop either as a result of inadequate dietary intake of niacin or tryptophan, or secondary to conditions that interfere with absorption $[9,10]$ Medications can also cause niacin deficiency, for example, patients with Parkinson syndrome treated with L-dopa is said often to suffer from niacin deficiency [11]. 
Despite the serious consequences of niacin and NAD depletion, these substances are hardly ever measured in the clinic. Niacin deficiency is usually assumed by the appearance of symptoms of pellagra and, occasionally, by the determination of metabolites of niacin excreted in the urine $[12,13]$. Pellagra is characterised by photosensitivity, diarrhoea, dermatitis and dementia. However, it is important to note that even in the absence of a diagnosis of pellagra, niacin deficiency may have effects on neuropsychiatric functioning $[9,10]$. Symptoms such as irritability, poor concentration, memory problems, anxiety, fatigue, restlessness, apathy, sleep disturbances, depression and dementia may result from niacin deficiency without it being recognised as such $[9,10]$.

The possibility of undiagnosed niacin deficiency underlying neuropsychiatric symptoms or disorders such as dementia is perhaps more pertinent in patients with poor dietary intake and in conditions with malabsorption. However, niacin, despite being classified as a vitamin, can also be synthesized in the body which probably offers a degree of protection in conditions of dietary insufficiency. De novo synthesis of niacin and NAD, as described in later paragraphs, increases in conditions marked by pro-inflammatory activity and is suppressed by substances with anti-inflammatory activity. The hypothesis is put forward that patients with poor dietary intake, who are treated with antidepressants, are at risk of developing niacin/NAD deficiency with possible development of the associated neuropsychiatric symptoms.

\section{Sources of niacin and NAD}

Niacin (nicotinamide and nicotinic acid) is abundant in fish, eggs, dairy products and in some vegetables [1]. Although meat is considered poor in niacin it contains NAD and NADP that 
are cleaved by mucosal enzymes and by nicotinamidase in the GIT to form nicotinamide and nicotinic acid, respectively, which are then absorbed into the circulation [1].

Both nicotinamide and nicotinic acid can be metabolised to NAD, but the pathways differ. Nicotinamide is metabolised to the pyridine nucleotide merely by reacting with phosphoribosyl-1-pyrophosphate. Nicotinic acid, however, must react with 5phosphoribosyl-1-pyrophosphate to form the nicotinic acid mononucleotide, which then condenses with ATP to form the nicotinic acid analogue of NAD, which is subsequently converted to NAD by a reaction with glutamine and ATP $[1,14,15]$.

Although niacin is generally described as a vitamin, niacin and NAD can also be synthesized in the body. See Figure 1. Tryptophan is the major substrate for the de novo synthesis of NAD. In the de novo pathway, synthesis from tryptophan occurs as part of the kynurenine pathway in the so-called tryptophan-nicotinamide pathway. NAD can also be generated by recycling degraded NAD components such as nicotinamide, nicotinic acid and nicotinamide riboside in the so-called salvage pathways $[6,16]$.

Although it has in the past been assumed that, under normal conditions, only about $2 \%$ of niacin, and by implication NAD, is derived from de novo synthesis [17], there are indications that the contribution is of much greater importance, even in normal healthy populations. Recent research from Japan reported nicotinamide synthesised from tryptophan in normal healthy Japanese to be almost equal to that from dietary intake [16]. Synthesis from tryptophan has even been suggested as the primary source of NAD in the body [14]. 


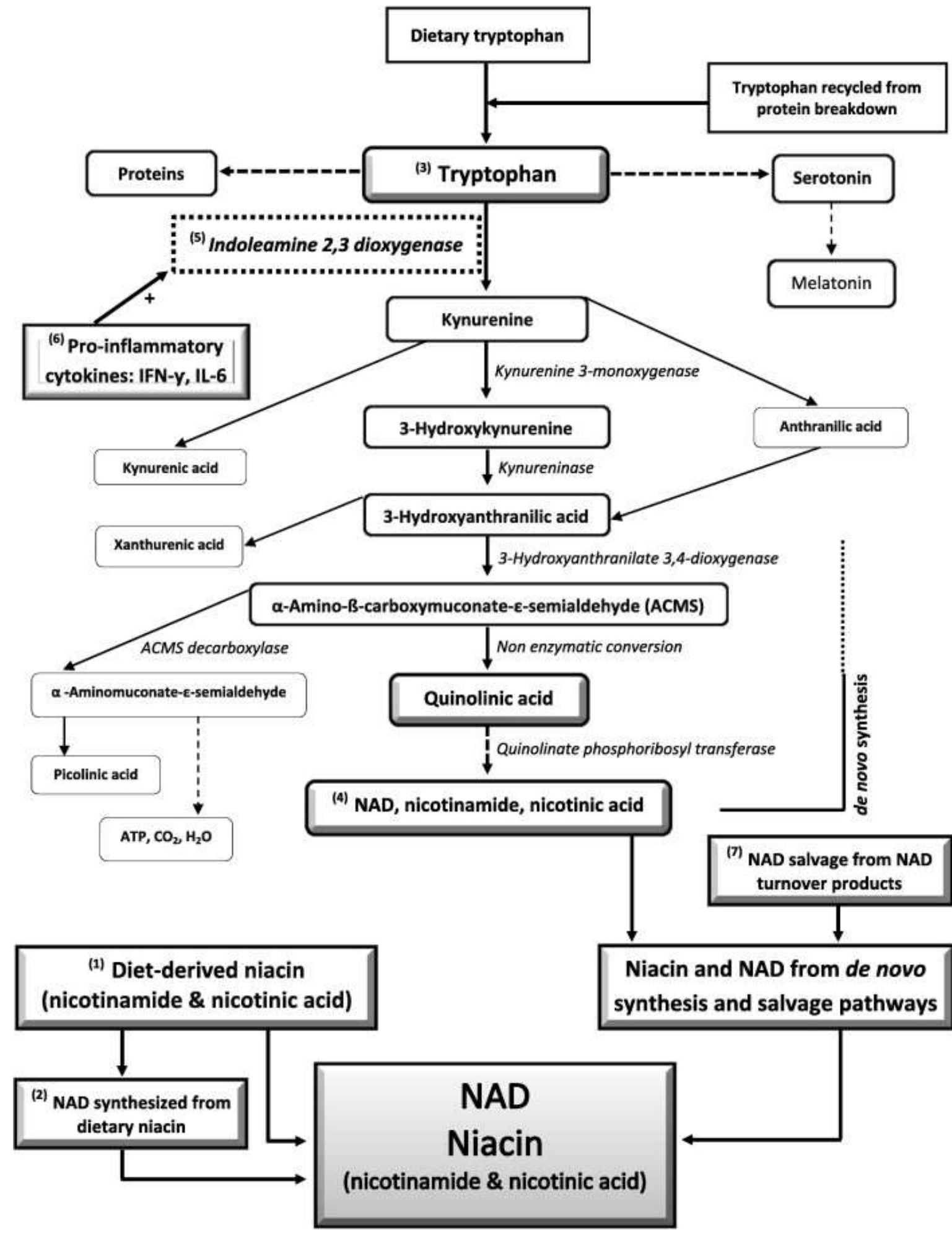

Figure 1: Sources of Niacin and NAD. Niacin (nicotinamide and nicotinic acid) is the dietary precursor for the synthesis of NAD (1 \& 2). Niacin and NAD can also be synthesized from tryptophan in the kynurenine pathway $(3 \rightarrow 4)$. Indoleamine 2,3 dioxygenase (IDO) is the rate-limiting enzyme for the de novo synthesis of niacin and NAD in the kynurenine pathway (5). IDO induction is regulated by pro-inflammatory cytokines such as IL-6 and IFN-gamma (6). NAD can also be salvaged from its turnover products (7). 


\section{Pro-inflammatory activity and the kynurenine pathway (de novo synthesis of niacin}

and NAD)

In the absence of sufficient dietary-derived niacin, tryptophan oxidative degradation in the kynurenine pathway remains the only metabolic route for the de novo synthesis of NAD and niacin [14]. The rate limiting step of tryptophan oxidation in the kynurenine pathway occurs under influence of the inducible enzyme indoleamine 2,3-dioxygenase (IDO) and is not limited by a decrease in tryptophan levels [18]. Pro-inflammatory cytokines are the driving force of IDO-activity. The main cytokines for the induction of IDO are the pro-inflammatory cytokines interferon-gamma (IFN- $\gamma$ ) [19] in the periphery and interleukin-6 (IL-6) in the central nervous system, but other pro-inflammatory cytokines may also have an influence [20]. De novo synthesis increases dramatically in chronic inflammatory conditions [21]. This inflammation-induced increase in activity in the kynurenine pathway may cause a drain on tryptophan levels that may reduce the availability of tryptophan for the biosynthesis of serotonin and melatonin $[18,22]$. In addition, excessive increases in activity in the kynurenine pathway may lead to the accumulation of the neurotoxin quinolinic acid, the direct precursor for NAD synthesis [14,23]. Nicotinamide levels increase in parallel with increases in quinolinic acid up to levels where saturation of the enzyme quinolinate phosphoribosyl transferase occurs. Toxic accumulation of quinolinic acid may occur with higher levels [24].

\section{Inhibition of the kynurenine pathway}

As the activity of pro-inflammatory cytokines is the main driving force behind increased tryptophan degradation in the kynurenine pathway, any factor that suppresses inflammatory cytokine activity, e.g. the synthesis of IFN-gamma in the periphery and IL-6 in 
the central nervous system, will counter the tryptophan drain. However, several beneficial effects have been described to inflammation-induced tryptophan depletion, including tryptophan depletion as a mechanism to starve pathogens and cancer cells and to facilitate immune tolerance [14]. In addition, inhibition of the activity of the kynurenine pathway will lower de novo synthesis of NAD that could, in the absence of adequate dietary supply of NAD precursors such as niacin, lead to intracellular NAD depletion. The effects of NAD depletion are widespread including reduced cell viability, compromised NAD-dependent SIRT1 activity and impaired central nervous system function [4].

\section{Anti-inflammatory actions of antidepressant medication}

Antidepressants have been reported to successfully control the immune reactivity and cytokine responses in depressed patients [25], and may be of benefit to patients with depression due to general medical conditions and depression induced by the therapeutic use of pro-inflammatory cytokines $[26,27]$. Antidepressants can suppress the secretion of pro-inflammatory cytokines, but other effects also exist through which antidepressants could counteract the effects of pro-inflammatory cytokines, including stimulation of the release of cytokine antagonists such as IL-1 receptor antagonist, and by acting as inhibitors of pro-inflammatory mediators such as cyclo-oxygenase [28].

Antidepressants have been shown to affect at least two types of immune cells: a) glial cells, which are the major immune cells responsible for inflammation in the brain and Thelper 1 cells (TH1) [29]. Some types of anti-depressants (e.g. imipramine, fluvoxamine and reboxetine) have been shown to inhibit activated glial cells [28]. In addition, diverse antidepressants belonging to different families, such as imipramine, venlafaxine, fluoxetine, 
clomipramine, sertraline and trazodone, consistently reduce the IFN- $\gamma /$ IL-10 ratio through their actions on TH1 cells [30]. Thus, antidepressants may exert their anti-inflammatory effects by decreasing pro-inflammatory cytokine levels (e.g. IFN- $\gamma$ ) or by increasing antiinflammatory cytokine levels (e.g. II-10) [29].

The mechanism by which antidepressants effect immune cells is widely accepted to involve upregulation of intracellular cAMP levels through the CAMP- protein kinase A pathway, possibly by suppressing phosphodiesterase catalyzed breakdown of CAMP $[28,29]$. Adjunctive treatment with anti-inflammatory agents may augment the therapeutic efficacy of anti-depressant medication. This suggests that antidepressants may act on downstream neurochemical consequences of elevated immune-inflammatory signaling, rather than exerting direct effects on signaling activity [31].

\section{Niacin and NAD+ levels in patients with poor dietary intake}

Eating disorders are often characterized by malnutrition [32] and it is feasible to expect the dietary intake of niacin to be compromised. Although a number of cases of pellagra have been described in association with anorexia nervosa [10,33], niacin or NAD levels have not been documented. However, nutritional deficiencies in psychiatric patients are not limited to those with eating disorders and nutritional supplementation has, in addition to patients with eating disorders, been suggested for individuals suffering from depression, bipolar disorder, schizophrenia, anxiety disorders, attention deficit/attention deficit hyperactivity disorder, autism and certain addictions [34]. Nutritional deficiencies are, for instance, relatively common in depressed patients where poor food choices may contribute to a 
deficit in those amino acids needed for the synthesis of essential neurotransmitters such as serotonin and noradrenalin [34].

Amino acids and the $B$ vitamins are amongst the most common nutritional deficiencies described for patients with mental disorders [34]. In contrast to other B vitamins, niacin levels are generally not included in nutritional surveys on psychiatric patients and published values do not exist. Nevertheless, it seems reasonable to assume a dietary deficit of niacin in patients with a general nutritional deficiency. At variance with essential amino acids and most vitamins, niacin, and by implication NAD are not solely dependent on dietary intake of niacin. It can also, as described in previous paragraphs, be synthesized in the body through the oxidative metabolism of tryptophan. The rate of this de novo synthesis of niacin and NAD in the kynurenine pathway of tryptophan metabolism depends on the presence of proinflammatory mediators.

\section{Conditions associated with increased activity in the kynurenine pathway}

Increased tryptophan degradation in the kynurenine pathway is reported for many diseases marked by increased pro-inflammatory activity, including various infections, autoimmune disorders, malignancies and neurodegenerative disorders [21]. Above normal levels of proinflammatory cytokines have also been reported for many psychiatric conditions [35]. Although, as yet no clear picture exists with regard to the cytokine profile in eating disorders, indications are that pro-inflammatory cytokines such as IL-1, IL-6, TNF $\alpha$ and IFN-y, may be instrumental in the pathogenesis of eating disorders [36]. In contrast to eating disorders, the link between many other psychiatric disorders, especially depression, and pro-inflammatory activity is well-known. [35,37]. 
It seems reasonable to assume that the presence of pro-inflammatory cytokines in these patients will stimulate de novo synthesis of NAD and its precursors and that it may even compensate for possible deficits in the dietary intake of niacin. This increase in de novo synthesis as a result of pro-inflammatory activity could, however, be suppressed by the antiinflammatory actions of antidepressants.

\section{In summary}

Patients who develop nutritional deficiencies as a result of poor dietary intake could potentially suffer from niacin deficiency and NAD depletion. However, de novo synthesis in the kynurenine pathway, due to induction of the enzyme IDO by pro-inflammatory cytokines such as interferon-gamma may, at least partially, compensate for the impaired dietary intake. Antidepressants have an anti-inflammatory effect, including reduction of interferongamma and therefore inhibition of IDO [38], the rate-limiting enzyme of the kynurenine pathway through which tryptophan is metabolized. In theory, this could account for increased serotonin as more tryptophan becomes available for serotonin synthesis. However, the downside may be that less NAD and niacin are synthesised downstream, which could exacerbate common psychiatric problems.

Niacin supplementation in patients who are known to have an inadequate diet or who have absorption problems and are started on antidepressant medication would provide the necessary building blocks for NAD synthesis. Indications are that niacin supplementation not only restores the NAD levels [4] but also has a feedback mechanism that decreases the 
tryptophan drain [39]. However, care should be taken with the prescribed dosages as excessive intake of niacin may lead to toxic blood levels [40].

We therefore propose that niacin supplementation be considered in patients with inadequate diets who are treated with antidepressants. We believe that if this does not happen, a subclinical niacin deficiency may result, which would be difficult to detect as it would cause the same symptoms of the original illness (e.g. depression). Niacin deficiency should be considered and ruled out in all patients with treatment-resistant depression, who have a poor response to antidepressants. This is potentially a cost-effective and easy intervention, which could be examined in a randomized controlled trial.

\section{Conflict of interest}

None

\section{References:}

1. Bogan KL, Brenner C. Nicotinic acid, nicotinamide, and nicotinamide riboside: $A$ molecular evaluation of $\mathrm{NAD}^{+}$precursor vitamins in human nutrition. Annu Rev Nutr 2008;28:115-130.

2. Murray MF. Nicotinamide: An oral antimicrobial agent with activity against both mycobacterium tuberculosis and human immunodeficiency virus. Clin Infect Dis $2003 ; 36: 453-460$.

3. Khan JA, Forouhar F, Tao X, Tong L. Nicotinamide adenine dinucleotide metabolism as an attractive target for drug discovery. Expert Opin Ther Targets 2007;11(5):695705. 
4. Braidy N, Guillemin GJ, Grant R. Effects of Kynurenine Pathway Inhibition on NAD Metabolism and Cell Viability in Human Primary Astrocytes and Neurons. Int J Tryptophan Res 2011;4:29-37.

5. Essa MM, Subash S, Braidy N, Adawi S, Lim CK, Manivasagam T, Guillemin G. Role of NAD+, Oxidative Stress, and Tryptophan Metabolism in Autism Spectrum Disorders. Int J Tryptophan Res 2013;6:15-28.

6. Lin S, Guarente L. Nicotinamide adenine dinucletide, a metabolic regulator of transcription, longevity and disease. Current opinion in Cell Biology 2003;15:241246.

7. Sasaki Y, Araki T, Milbrandt J. Stimulation of Nicotinamide Adenine Dinucleotide Biosynthetic Pathways Delays Axonal Degeneration after Axotomy 2006;26(33):8484-8491.

8. Rex A, Schickert R, Fink H. Antidepressant-like effect of nicotinamide adenine dinucleotide in the forced swim test in rats. Pharmacology Biochemistry and Behavior 2004;77(2):303-7.

9. Amanullah S, Seeber $\mathrm{C}$. Niacin deficiency resulting in neuropsychiatric symptoms: a case study and review of literature. Clinical Neuropsychiatry 2010;7(1):10-14.

10. Prousky JE. Pellagra may be a rare secondary complication of anorexia nervosa: a systematic review of the literature. Altern Med Rev 2003;8(2):180-185.

11. Bender DA, Earl CJ, Lees AJ. Niacin depletion in Parkinsonian patients treated with Ldopa, benserazide and carbidopa. Clin Sci 1979;56:89.

12. Seal AJ, Creeke PI, Dibari F, Cheung F, Kyroussis E, Semedo P, van den Briel T. Low and deficient niacin status and pellagra are endemic in postwar Angola. Am J Clin Nutr 2007;85:218-224. 
13. Monteiro JP, da Cunha DF, Filho DC, Silva-Vergara ML, dos Santos VM, da Costa JC et al. Niacin metabolite excretion alcoholic pellagra and AIDS patients with and without diarrhea. Nutrition 2004;20:778-782.

14. Moffet JR, Namboodiri MA. Tryptophan and the immune response. Immunology and Cell Biology 2003;81:247-265.

15. Scientific Committee on Food, European Commission, Health \& Consumer Protection Directorate-General. Opinion of the Scientific Committee on Food on the Tolerable Upper Intake Levels of Nicotinic Acid and Nicotinamide (Niacin). SCF/CS/NUT/UPPLEV/39 6 May 2002.

16. Fukuwatari T, Shibata K. Nutritional aspect of tryptophan metabolism. International Journal of Tryptophan Research 2013;6:3-8.

17. Murray MF. Tryptophan depletion and HIV infection: a metabolic link to pathogenesis. Lancet Infect Dis 2003;3:644-52.

18. Chen Y, Guillemin GJ. Kynurenine pathway metabolites in humans: disease and health states. International Journal of tryptophan research 2009;2:1-19.

19. Fuchs D, Moller AA, Reibnegger $G$ et al. Increased endogenous interferon-gamma and neopterin correlate with increased degradation of tryptophan in human immunodeficiency virus type 1 infection. Immunol Lett 1991;28:207-211.

20. Boasso A, Shearer G. How does indoleamine 2,3 dioxygenase contribute to HIVmediated immune dysregulation. Current Drug Metabolism 2007;8:217-223.

21. Schroeksnadel K, Sarcletti M, Winkler C et al. Quality of life and immune activation in patients with HIV infection. Brain Behav Immun 2008;22:881-889. 
22. Murray MF, Langan M, MacGregor RR. Increased plasma tryptophan in HIV-infected patients treated with pharmacologic doses of nicotinamide. Nutrition 2001;17:654656.

23. Guillemin GJ. Quinolinic acid, the inescapable neurotoxin. FEBS J 2012;279:13561365.

24. Bipath P. Dysregulation of tryptophan metabolism in HIV/AIDS. Thesis. University of Pretoria 2014.

25. Yirmiya R, Weidenfeld J, Pollack Y, Morag M, Morag Y, Avitsur R, Barak O, Reichenberg A, Cohen E, Shavit Y, Ovadia H. Cytokines, depression due to a general medical condition and antidepressant drugs. Kluwer Academic/ Plenum Publishers, New York, 1999, pp283-307.

26. Katon W, Sullivan MD. Depression and chronic medical illness. J Clin Psychiatry 1990;51:3-11.

27. Levenson JL, Fallon HJ. Fluoxetine treatment of depression caused by interferon alpha. Am J Gastroenterol 1993;88:760-761.

28. Leonard BE. Impact of inflammation on neurotransmitter changes in major depression: an insight into the action of antidepressants. Prog Neuropsychopharmacol Biol Psychiatry 2014;48:261-7.

29. Hashioka S, McGeer PL, Monji A, Kanba S. Anti-inflammatory effects of antidepressants: possibilities for preventives against Alzheimer's disease. Central Nervous System Agents in Medicinal Chemistry 2009;9:12-19.

30. Frick LR, Rapanelli M. Antidepressants: influence on cancer and immunity? Life Sci 2013;92(10):525-32. 
31. McNamara RK, Lotrich FE. Elevated immune-inflammatory signaling in mood disorders: a new therapeutic target? Expert Rev Neurother 2012;12(9):1143-61.

32. Patrick L. Eating disorders: a review of the literature with emphasis on medical complications and clinical nutrition. Altern Med Rev 2002;7:184-202.

33. Jonathan E. Prousky. Treating Dementia with Vitamin B3 and NADH. JOM 2011;26(4):163-174.

34. Sathyanarayana Rao TS, Asha MR, Ramesh BN, Jagannatha Rao KS. Understanding nutrition, depression and mental illnesses. Indian J Psychiatry 2008;50(2):77-82.

35. Viljoen, M, Panzer, A. Non-termination of sickness behavior as precipitating factor for mental disorders. Medical Hypotheses 2005;64(2):316-329.

36. Corcos M, Guilbaud O, Paterniti S, Moussa M, Chambry J, Chaouat G, Consoli SM. Involvement of cytokines in eating disorders: a critical review of the human literature. Psychoneuroendocrinology 2003;28(3):229-49.

37. Maes M. Major depression and activation of the inflammatory response system. Adv Exp Med Biol 1999;461:25-46.

38. Apalset EM, Gjesdal CG, Ueland PM, Midttun $\varnothing$, Ulvik A, Eide GE, Meyer K, Tell GS. Interferon (IFN)- $\gamma$-mediated inflammation and the kynurenine pathway in relation to bone mineral density: the Hordaland Health Study. Clin Exp Immunol $2014 ; 176(3): 452-60$.

39. Taylor EW. The oxidative stress-induced niacin sink (OSINS) model for HIV pathogenesis. Toxicology 2010;278(1):124-13040.

40. Guyton JR, Bays HE. Safety considerations with niacin therapy. Am J Cardiol 2007;99:22C-31C. 